\section{Welcome to the incredible world of MAP Kinases}

Jonas Cicenas

CALIPHO group, Swiss Institute of Bioinformatics, Geneva, Switzerland and MAP Kinase Resource, Bern, Switzerland

First of all, I would like to welcome everybody to our new journal, and that includes members of the Editorial Board, authors, and you the readers. I am happy to tell you that our Editorial Board is made up of well-known experts in various aspects of MAP kinase research and they will be contributing their knowledge and expertise to this exciting new project.

MAP Kinase is the first journal to concentrate specifically on the Mitogen-activating protein kinase family of protein kinases. MAP kinases are serine/threonine protein kinases that respond to extracellular stimuli and regulate various cellular activities, such as apoptosis, gene expression, mitosis, differentiation and immune responses, to name just a few. The MAP kinase family consists of four major subfamilies of related proteins (Erk1/2, JNK, p38 and Erk5) that make up interconnected signal transduction cascades activated by external stimuli, such as growth factors, stress, cytokines and inflammation. Deregulation of these pathways can lead to various disorders, such as cancer, autoimmune diseases, neurodegenerative diseases, cardiovascular diseases, inflammation, etc. This means that almost any area of biology, biomedicine and clinical science involved to a greater or lesser degree in this type of research will include MAP kinases. Skeptics have told me that MAP kinases might be too narrow a field for one journal, an argument that I completely disagree with. Anyone is welcome to suggest a biological process in eukaryotes in which no MAP kinase is involved and I promise to write an erratum to this article! There is an incredible number of publications on MAP kinases. If we only look for papers published over the last year, a PubMed search for Erk, restricted to title only, results in more than 600 publications, $J N K$ in more than $300, p 38$ in more than $500, M A P K$ in more than 600, map kinase in more than 100, and mitogen activated protein kinase in more than 200. Even if we consider any possible overlap, this is an incredible number. I really hope that publications in our journal will soon join and add to them.

The enormous variety of the subject areas in which MAP kinases are involved will be reflected in the broad scope of the MAP Kinase journal. Biochemistry, protein structure, cell biology, molecular biology, pharmacology, neurobiology, molecular endocrinology, molecular oncology, clinical oncology, developmental biology, physiology and proteomics are all considered major topics. However, no good quality paper dealing with MAP kinases will be turned down by our journal. We expect to receive original research articles, short reports, case reports, database articles, reviews and minireviews. Book reviews would also be welcome. We also hope that members of our editorial board will help organize some special issues. These could be dedicated to one of the subfamilies, or to a specific biological process, disease or organism. The journal will be published in Open Access format. In my opinion, the fact that everybody and anybody has access to scientific data is the most important achievement of our century. It is really great to know that important organizations like the NIH and the NCBI also support the idea of Open Access. But online publishing also offers other important possibilities. One of the problems in conventional publishing is the long time one has to wait for submitted manuscripts to complete peer review and go through the publication process. We will do our best to work to a 20-30 day time scale from submission to end publication (in case of acceptance). Having a quick turn around and ensuring speedy publication is very important for original research papers. In addition, there are some researchers who conduct important research but who cannot adequately highlight the positive sides of their manuscripts because of linguistic barriers or inadequate discussion and/or interpretation of
Correspondence: Jonas Cicenas, CALIPHO group, Swiss Institute of Bioinformatics, Geneva, Switzerland and MAP Kinase Resource, Bern, Switzerland.

E-mail: j.cicenas@mapkinases.eu

This work is licensed under a Creative Commons Attribution NonCommercial 3.0 License (CC BYNC 3.0).

(C) Copyright J. Cicenas, 2013

Licensee PAGEPress, Italy

MAP Kinase 2013; 2:el

doi:10.4081/mk.2013.e1

their results. We are happy to help these scientists! Our reviewers will help with the structure of the manuscripts and they will point out any possible linguistic problems. PAGEPress also offers professional assistance to nonEnglish speaking authors. Remember, we also fight plagiarism! All articles will be checked for plagiarism so that we can protect authors al over the world.

And, last but not least, the journal will be linked to the MAP Kinase Resource (http://www.mapkinases.eu), the web-based resource and database dedicated to various aspects of MAP kinase research and education. One of the services provided by the MAP Kinase Resource is to integrate the journal into the website. We also encourage our reviewers, authors and readers to join the map kinase forum at http://community.mapkinases.eu where you can discuss publications, research methods and other aspects of MAP kinase research. In launching MAP Kinase, we are hopeful that we can attract the best, most novel and most interesting manuscripts in the field of MAP kinases. To all those future contributors to our journal, we promise that, once your work is accepted, it will quickly be distributed all over the world. Let's work together to make the world of MAP kinases a better place to be!

Sincerely yours,

Jonas Cicenas The Editor-in-Chief 